\title{
Recurrent Hepatocellular Carcinoma After Living Donor Liver Transplantation: A Preventable Problem or an Acceptable Risk?
}

\author{
Christopher J. Sonnenday, MD, MHS \\ Department of Surgery, University of Michigan, Ann Arbor, MI
}

Hepatocellular carcinoma (HCC) remains one of the most important surgical diseases worldwide, as it is the $3 \mathrm{rd}$ deadliest malignancy accounting for more than 700,000 deaths worldwide. ${ }^{1}$ Estimated new cases number close to 800,000 cases annually, and in many parts of the world HCC incidence is increasing faster than any other cancer. ${ }^{2}$ Treatment strategies for HCC are also evolving quickly as new local and systemic therapeutic innovations develop. The heterogeneous treatment options can be confusing to patients and providers alike; often such protean approaches to a single disease suggest we have not arrived yet upon the most favorable strategy. For many patients, liver transplantation appears to represent that optimal approach-allowing definitive resection of the malignancy while treating underlying chronic liver disease. However, the application of liver transplantation to patients with HCC has been limited by donor organ scarcity, the tremendous resources required, and the significant risks inherent to the procedure.

In this edition of the journal, Taketomi and colleagues report on the downstream consequences and oncologic principles involved with extending criteria for transplantation of HCC using living liver donation. ${ }^{3}$ Living donor liver transplantation (LDLT) has several notable advantages in its application to HCC including: avoidance of prolonged waiting times for deceased donor organs, allowing elective timing of transplantation to correlate with pretransplant neoadjuvant and downstaging strategies, preventing patients with advanced malignancies from competing with patients with benign causes of liver failure awaiting transplant, and providing organ replacement therapy in parts of the world where brain death and deceased donation are less commonly accepted. As LDLT is offered to patients with more advanced

(C) Society of Surgical Oncology 2010

Published Online: 30 April 2010

C. J. Sonnenday, MD, MHS

e-mail: csonnend@umich.edu
HCC, careful attention to posttransplant outcomes is required, including the appropriate management of recurrent disease.

The group from Kyushu University Hospital describes their experience with a cohort of 101 adult patients treated for HCC with LDLT, focusing on the management of 17 patients who developed recurrent HCC after transplant. All but 1 of the patients with recurrent disease were transplanted for tumors beyond Milan criteria, which have become broadly accepted as the appropriate criteria for recipient selection with relatively few exceptions. The group described a liberal approach to patient selection, with extrahepatic disease and gross vascular invasion stated as the only hard contraindications to transplantation. Tumor burden (size or number of tumors) was not considered as criteria for selection, except to stratify patients for adjuvant systemic chemotherapy. The authors report the use of a rather robust immunosuppression regimen, using 3 drugs and induction with an IL-2 receptor antagonist. The reported survival in patients without tumor recurrence is outstanding, and a testament to the Kyushu group's technical prowess with LDLT.

The authors took an aggressive approach to recurrent HCC posttransplant. Multimodality therapy including surgical resection, radiofrequency ablation, radiation therapy, and systemic chemotherapy was fastidiously employed to attack areas of recurrent disease. Despite relatively early recurrences (mean time from transplant 12.9 months, earliest in the 2nd month), 9 of the 17 recurrent patients underwent 1 or more surgical resections, and these select individuals were able to achieve overall patient survival that was similar to patients without tumor recurrence, though many of the patients appear to have residual disease. Patients who were not eligible for surgical resection of recurrent disease had uniformly poor outcomes, with all but 1 dead of recurrent disease by 26 months post-transplant. Systemic chemotherapy alone, which did not include sorafenib, did not appear to have an impact on either the development of recurrent disease or on overall survival. 
The present study appears to legitimize an aggressive, and often surgical, approach to recurrent HCC post-transplant. Select patients with solitary recurrences or oligometastatic disease may achieve reasonable overall survival, and the Kyushu report adds to the evidence that transplant patients deserve aggressive approaches to malignant disease, despite the challenges of their medical complexity and immunosuppressed state. However, hidden between the lines of the present study are important implications about the role of liver transplantation, and specifically LDLT, in the management of advanced HCC. If one was to take an intention-to-treat approach to the patients in the Kyushu experience with HCC beyond Milan criteria, did LDLT add additional life-years to this cohort?

As therapies for HCC improve, the treatment-modified natural history of advanced HCC is becoming more unpredictable. Patients without gross extrahepatic disease can have a protracted course of disease, with sequential episodes of liver-directed therapy including hepatic resection, radiofrequency ablation, transarterial chemoembolization, radionuclide embolization, and other ablative techniques (percutaneous ethanol injection, microwave ablation, cryotherapy, etc.). With the exception of surgical resection, all of these treatments for advanced HCC are often seen as palliative, and downstaging to a period of disease stability that falls within Milan criteria is often the goal, allowing transplantation to be at least considered as an attempt at definitive curative treatment. However, many patients with large tumor burdens can have reasonable survival on treatment, particularly if any associated underlying chronic liver disease remains compensated.

So, is transplant always the optimal therapy? Clearly in settings where deceased donors supply the majority of organs for transplant, directing organs to patients with advanced HCC would deprive patients with benign causes of liver disease, who on average have better predicted longterm outcomes, of precious opportunities at life-saving therapy. However, what about when the potential recipient is able to produce a living donor candidate? Do the high standards for acceptable outcomes after transplantation get loosened somewhat with LDLT? After all, presumably no other recipient on the list is being deprived of an opportunity for transplant as the living donor is directed to the individual recipient (altruistic donors as an obvious but rare exception). Besides ensuring that donor and recipient are properly counseled about the increased risk of recurrent cancer and diminished long-term outcomes, and with the paramount importance of providing a safe donor and recipient operation, is the transplant community obligated to restrict LDLT to the most appropriate candidates?

Clearly the strategy described by the Kyushu groupproviding LDLT to transplant patients with advanced HCC regardless of tumor burden, and using aggressive surveillance and multimodality therapy to address recurrent disease-represents a paradigm shift from other strategies that have been vetted and debated among the transplant and oncology communities. John Wong and others popularized the strategy of resection for $\mathrm{HCC}$, saving transplantation as salvage therapy for patients with recurrent disease or decompensated liver function. ${ }^{3}$ Advocates of downstaging protocols for HCC typically describe a period of disease stability following pretransplant therapy to allow some reassurance against particularly aggressive tumor biology. ${ }^{4}$ Both of these approaches exploit 1 of the major advantages of neoadjuvant approaches to the surgical treatment of malignancy, specifically, providing a period of treatment and observation to observe the biologic behavior of the tumor. Patients who have significant disease progression on neoadjuvant therapy have poor outcomes and would likely not have been helped by aggressive surgical resection or transplantation. Bypassing such an observation period in the setting of multifocal or locally advanced tumors risks transplanting patients with systemic disease, as the pattern of recurrence in the Kyushu experience suggests.

Moving forward, it is likely that the pendulum will continue to swing gradually in favor of cautiously expanding beyond the Milan criteria for liver transplantation of HCC. Improved understanding of tumor behavior in response to liver-directed therapy, the incorporation of adjuvant systemic therapies, and enhanced biologic markers of tumor behavior will hopefully allow refined selection of appropriate transplant candidates beyond the constraints of tumor size and number. As more patients with HCC are transplanted, a refined understanding of the management of post-transplant recurrence will also be necessary, and the Kyushu group's aggressive multidisciplinary approach offers important lessons in this area. However, equally important is an evolving understanding of the appropriate indications for liver transplantation as a definitive therapy for HCC. Paramount in our minds should be our obligation to the optimal use of precious donor organs, and that responsibility should be no less stringent for living donors.

\section{REFERENCES}

1. Garcia M, Jemal A, Ward EM, Center MM, Hao Y, Siegel RL, et al. Global cancer facts \& figures 2007. Atlanta, GA: American Cancer Society; 2007.

2. World Health Organization. Available: www.who.int; 2009 [cited September 1, 2009].

3. Poon RT, Fan ST, Lo CM, Liu CL, Wong J. Long-term survival and pattern of recurrence after resection of small hepatocellular carcinoma in patients with preserved liver function: implications for a strategy of salvage transplantation. Ann Surg. 2002;235:373-82.

4. Yao FY, Kerlan RK Jr, Hirose R, Davern TJ 3rd, Bass NM, Feng $\mathrm{S}$, et al. Excellent outcome following down-staging of hepatocellular carcinoma prior to liver transplantation: an intention-to-treat analysis. Hepatology. 2008;48:819-27. 